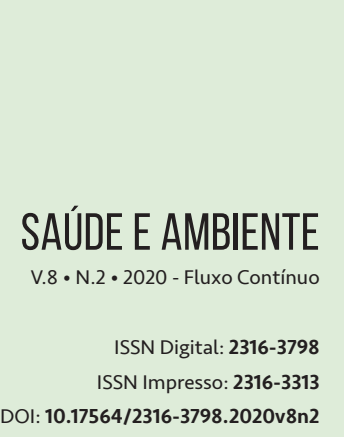

DOI: $10.17564 / 2316-3798.2020 v 8 n 2$

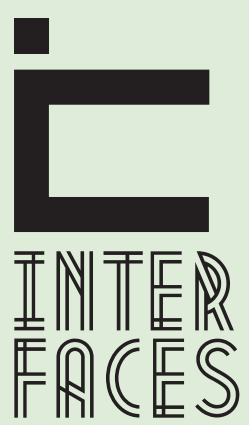

CIENTÍFICAS

\section{AVALIACC̄̃O DO ABASTECIMENTO DE ÁGUA DO MARANHÃO ATRAVÉS DO SISTEMA NACIONAL DE INFORMAÇ̃ES SOBRE SANEAMENTO}

\section{EVALUATION WATER SUPPLY OF THE MARANHÃO THROUGH THE NATIONAL SANITATION INFORMATION SYSTEM}

\section{EVALUACIÓN DEL ABASTECIMIENTO DE AGUA DEL MARANHÃO A TRAVÉS DEL SISTEMA NACIONAL DE INFORMACIÓN SOBRE SANEAMIENTO}

Marylin Fonseca Leal de Farias Wetters ${ }^{1}$ Solange da Silva Nunes Boni ${ }^{2}$

Nathalia Cunha Almeida Pinheiro ${ }^{3}$ Roni Cleber Boni ${ }^{4}$

\section{RESUMO}

O abastecimento de água possui sérios problemas de gestão no Brasil e no mundo. A prestação do serviço, quando de forma inadequada, gera impactos na área da saúde, economia e meio ambiente. Para uma melhor compreensão da situação do abastecimento de água tanto por gestores quanto por consumidores, foram criadas ferramentas com base das informações coletadas nas concessionárias, as quais visa o monitoramento da qualidade dos serviços prestados. Dentre os dados existentes para análise, alguns indicadores se destacam pela fácil interpretação e grande utilidade para a gestão pública, dentre eles: econômico-financeiros e administrativos, operacionais - água e de qualidade. 0 objetivo deste trabalho foi avaliar o sistema de abastecimento de água do estado do Maranhão a fim de criar subsídios técnicos para contribuir com a gestão do abastecimento de água através dos índices de Incidência das análises de cloro residual, Incidência das análises de turbidez e Incidência das análises de coliformes totais todos fora do padrão. Os dados utilizados como base para a avaliação foram disponibilizados pela Lei Federal No 11.445 , SNIS e pela CAEMA cujo período de análise compreendeu informações de 2007 a 2017. Observou-se variações de seus índices nos 10 anos avaliados, concluindo-se que muito ainda deverá ser feito para que o estado do Maranhão atinja uma boa qualidade na universalização do serviço de abastecimento de água urbana. Verificou-se que são necessárias algumas melhorias, no que se refere à minimização desses três indicadores, principalmente por estarem diretamente relacionados a saúde e bem-estar da população. 


\section{PALAVRAS-CHAVE}

Abastecimento Público, Maranhão, Saneamento Básico, Indicadores do SNIS.

\section{ABSTRACT}

The water supply has serious management problems in Brazil and in the world. The provision of the service, when inadequately, generates impacts in the area of health, economy and environment. For a better understanding of the water supply situation by both managers and consumers, tools were created based on the information collected in the concessionaires, which aims to monitor the quality of the services provided. Among the data available for analysis, some indicators stand out for the easy interpretation and great utility for public management, among them: economic-financial and administrative, operational - water and quality. The objective of this study was to evaluate the water supply system of the state of Maranhão in order to create technical subsidies to contribute to the management of water supply through the Incidence of residual chlorine analysis, Incidence of turbidity analyzes and Incidence of analysis of total coliforms all non-standard. The data used as a basis for the evaluation were made available by Federal Law No.11,445, SNIS and CAEMA, whose period of analysis included information from 2007 to 2017. Variations of their indexes were observed in the 10 years evaluated, be made so that the state of Maranhão reaches a good quality in the universalization of the urban water supply service. It has been found that some improvements are needed, as regards the minimization of these three indicators, mainly because they are directly related to the health and well-being of the population.

\section{KEYWORDS}

Public Supply, Maranhão, Basic Sanitation, Indicators of the SNIS.

\section{RESUMEN}

El suministro de agua tiene serios problemas de gestión en Brasil y en el mundo. La prestación del servicio, cuando es inadecuado, genera impactos en el área de la salud, la economía y el medio ambiente. Para una mejor comprensión de la situación del suministro de agua, tanto por parte de los administradores como de los consumidores, se crearon herramientas basadas en la información recopilada en los concesionarios, que tienen como objetivo monitorear la calidad de los servicios pres- 
tados. Entre los datos disponibles para el análisis, algunos indicadores se destacan por su fácil interpretación y gran utilidad para la gestión pública, entre ellos: económico-financiero y administrativo, operativo - agua y calidad. El objetivo de este estudio fue evaluar el sistema de suministro de agua del estado de Maranhão con el fin de crear subsidios técnicos para contribuir a la gestión del suministro de agua a través de la incidencia del análisis de cloro residual, la incidencia de los análisis de turbidez y la incidencia de Análisis de coliformes totales todos no estándar. Los datos utilizados como base para la evaluación fueron puestos a disposición por la Ley Federal No 11.445, SNIS y CAEMA, cuyo período de análisis incluyó información de 2007 a 2017. Se observaron variaciones de sus índices en los 10 años evaluados, Se debe hacer para que el estado de Maranhão alcance una buena calidad en la universalización del servicio de suministro de agua urbana. Se ha encontrado que se necesitan algunas mejoras en lo que respecta a la minimización de estos tres indicadores, principalmente porque están directamente relacionados con la salud y el bienestar de la población.

\section{PALABRAS-CLAVE}

Abastecimiento Público, Maranhão, Saneamiento, Indicadores del SNIS

\section{INTRODUCÇÃO}

O abastecimento de água possui sérios problemas de gestão no Brasil e no mundo. A prestação do serviço, quando de forma inadequada, gera impactos na área da saúde, economia e meio ambiente.

Para uma melhor compreensão da situação do abastecimento de água tanto por gestores quanto por consumidores, foram criadas ferramentas com base das informações coletadas nas concessionárias, as quais visam o monitoramento da qualidade dos serviços oferecidos.

O desempenho dos prestadores de serviços, o desenvolvimento de indicadores pelas agências reguladoras permite exercer pressões competitivas que estimula a melhoria do serviço prestado, auxiliando, inclusive, na tomada de decisões quanto aos investimentos a serem destinados aos municípios e sistemas (CORTON, 2003). No Brasil, a regulação do setor de saneamento é realizada por agências municipais ou estaduais.

De acordo com VEIGA e colaboradores (2013), para área de gerenciamento de ações de saneamento, os indicadores, quando são bem desenvolvidos, proporcionam uma visão mais ampla, fornecendo subsídios para a tomada de decisão dos gestores.

A água para consumo da população necessita passar por diversos processos para o alcance dos indicies de potabilidade os quais são realizados por meio de diversos níveis em estações de tratamento de água.

A Portaria de Consolidação n 5, publicada em 28 de setembro de 2017 (BRASIL, 2017), descreve as normas sobre as ações e os serviços de saúde do Sistema Único de Saúde e, ainda, enfatiza a correlação dos padrões de qualidade da água com a saúde pública. 
Segundo Von Sperling (1996), a qualidade da água depende das condições naturais da bacia hidrográfica, bem como, das interferências do homem nessa bacia. Porém, para que uma água tenha qualidade desejável deve-se verificar qual será as atividades previstas para o uso desta água.

Dentre os dados existentes para análise, através do Sistema Nacional de Informações sobre Saneamento (SNIS), alguns indicadores se destacam pela fácil interpretação e grande utilidade para a gestão pública, dentre eles: econômico-financeiros e administrativos, operacionais - água e de qualidade.

O objetivo deste trabalho foi avaliar o sistema de abastecimento de água do estado do Maranhão a fim de criar subsídios técnicos para contribuir com a gestão do abastecimento de água.

\section{MÉTODO}

O estado do Maranhão está localizado na Região Nordeste do país. Constitui-se de um importante polo portuário brasileiro, com atividades de exportação de minérios, soja e agronegócios.

Apesar das referidas atividades, cabe ressaltar que o rendimento nominal mensal domiciliar per capita é baixo, sendo da ordem de R\$ 605,00 reais, ocupando a posição de $27^{\circ}$ vigésimo sétimo do país, ou seja, o penúltimo estado no que se refere a este parâmetro de comparação (IBGE, 2018).

No último censo, o Maranhão apresentou população residente de 6.547 .789 habitantes, sendo distribuídos em 4.147.149 habitantes urbanos (63,3\%) e 2.427.640 habitantes rurais (36,7\%) (IBGE, 2010).

Destaca-se também que de acordo com o último Diagnóstico dos Serviços de Água e Esgotos 2017 divulgado pelo SNIS, somente 52,7\% (cinquenta e dois virgula setenta por cento) da população do estado possui atendimento total a água (SNIS, 2017).

A metodologia aplicada contemplou as análises (apenas aquelas fora do padrão) dos índices de incidência do cloro residual, assim como dos parâmetros de qualidade turbidez e coliformes totais, disponibilizados por meio do Diagnóstico dos Serviços de Água e Esgoto apresentados pelo Sistema Nacional de Informações sobre Saneamento e pela Companhia de Saneamento Ambiental do Maranhão em conformidade com as exigências da Lei Federal n 11.455/2007 (BRASIL, 2007).

Ressalta-se pela referida lei que a União é responsável pela elaboração, sob coordenação do Ministério das Cidades o Plano Nacional de Saneamento Básico (PLANSAB), instrumento de implementação da política federal de saneamento básico.

Ressalta-se ainda que no intervalo considerado entre os anos 2007 a 2011, as análises dos dados foram obtidas a partir das informações dos municípios constantes no SNIS, excluindo-se aqueles cujos os indicadores estavam dentro do padrão e aqueles que não apresentaram nenhuma informação.

\section{RESULTADOS}

A tabulação dos dados da análise da incidência de cloro residual, turbidez e coliformes totais fora dos padrões, nos períodos considerados entre 2007 a 2017, retratam que houveram picos extremos 
com relação a qualidade física, química e biológica da água dos municípios maranhenses, com destaque negativo para os anos de 2007, 2010 e 2011 (TABELA 1).

Vale observar que para o ano de 2009, não havia dados disponíveis dos parâmetros estudados para o estado do Maranhão junto a base de dados considerada na pesquisa.

Tabela 1 - Dados tabulados dos indicadores analisados no estado do Maranhão

\begin{tabular}{|c|c|c|c|}
\hline $\begin{array}{c}\text { Ano considerado } \\
\text { na análise }\end{array}$ & $\begin{array}{c}\text { Incidência das análises } \\
\text { de cloro residual fora do } \\
\text { padrão (\%) }\end{array}$ & $\begin{array}{c}\text { Incidência das análi- } \\
\text { ses de turbidez fora do } \\
\text { padrão (\%) }\end{array}$ & $\begin{array}{c}\text { Incidência das análises de } \\
\text { coliformes totais fora do } \\
\text { padrão (\%) }\end{array}$ \\
\hline 2007 & 28 & 5,34 & - \\
\hline 2008 & 7,2 & 4,4 & 34,8 \\
\hline 2010 & 42,8 & 21,7 & 86,1 \\
\hline 2011 & 95,4 & 92,2 & 2,07 \\
\hline 2012 & 2,56 & 3,02 & 4,7 \\
\hline 2013 & 2,68 & 3,13 & 8,03 \\
\hline 2014 & 4,74 & 3 & 7,14 \\
\hline 2015 & 5,64 & 2,5 & 7,3 \\
\hline 2016 & 7,76 & 2,9 & 5,26 \\
\hline 2017 & 2,07 & 4,24 & \\
\hline
\end{tabular}

Fonte: Ministério das Cidades - Secretaria Nacional de Saneamento Ambiental, Sistema Nacional de Informações sobre Saneamento - SNIS.

Pela análise das informações da tabela verifica-se que o parâmetro físico turbidez, nos anos de 2007 e 2011, apresentou índices elevados em grande parte dos municípios maranhenses, estando fora dos padrões exigidos pela Portaria de Consolidação nº 5 do Ministério da Saúde (BRASIL, 2017). Ressalta-se que para o ano de 2009 , os autores não encontraram nenhuma informação referente a dados qualitativos da água para o estado.

Já no ano de 2010, percebeu-se que 92,2\% dos municípios maranhenses avaliados apresentaram em valores de turbidez muito acima do máximo permitido por norma (FIGURA 1), de acordo com os dados disponibilizados pelo SNIS. 
Figura 1 - Resultado dos dados do parâmetro turbidez para o estado do Maranhão - período: 2007 a 2011

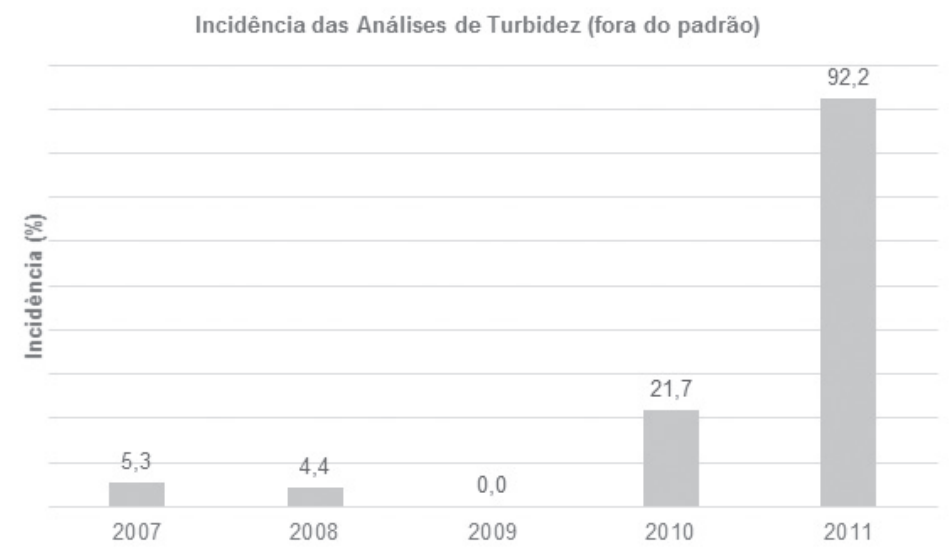

Fonte: SNIS (2019).

No entanto, de 2012 até 2017, poucos municípios maranhenses apresentaram turbidez fora da faixa estabelecida pelo Ministério da Saúde, com taxas de incidência variando de 2,5\% a 4,24\% (FIGURA 2).

Figura 2 - Resultado dos dados do parâmetro turbidez para o estado do Maranhão - período: 2012 a 2017

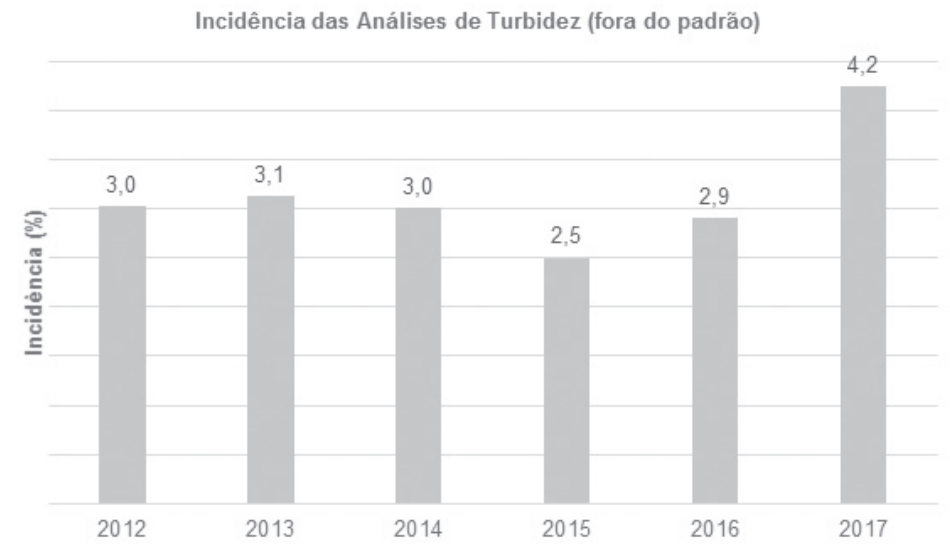

Fonte: SNIS (2019).

Com relação ao parâmetro microbiológico, nos anos de 2010 e 2011 houve muitos municípios, distribuindo água com a presença de coliformes totais, chegando a uma incidência de $86,1 \%$ de casos fora dos padrões. Segundo a norma do Ministério da Saúde, este parâmetro deve ser ausente em 100 $\mathrm{mL}$ de água tratada na saída do sistema de abastecimento (FIGURA 3). 
Figura 3 - Resultado dos dados do parâmetro coliformes totais para o estado do Maranhão - período: 2007 a 2011

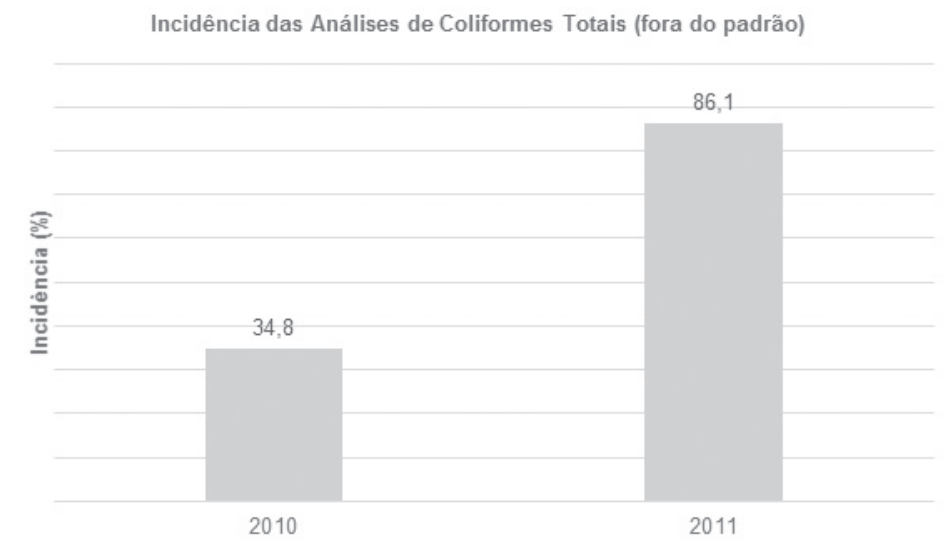

Fonte: SNIS (2019)

Porém, entre os anos de 2012 e 2017 houve um decréscimo da incidência de coliformes totais fora dos padrões nos municípios que forneceram os dados. No entanto, estes valores ainda são considerados altos, fator que pode evidenciar condições irregulares de qualidade da água distribuída em muitos municípios do Maranhão (FIGURA 4).

Figura 4 - Resultado dos dados do parâmetro Coliformes totais para o estado do Maranhão - período: 2012 a 2017

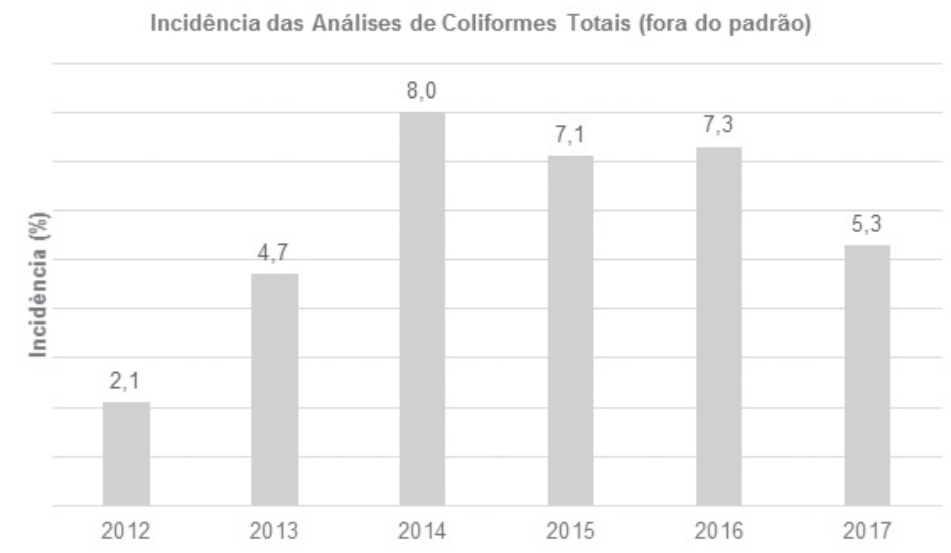

Fonte: SNIS (2019). 


\section{DISCUSSÃO}

Os resultados apresentados demonstram que há uma grande variabilidade do percentual dos valores obtidos da consulta ao SNIS para os anos analisados, comum a todos os parâmetros considerados.

Isto demonstra que muitos destes dados podem apresentar erros quando da sua concepção por parte dos municípios pertencentes ao estado do Maranhão, o que denotam que muitos destes podem estar inconsistentes.

Um exemplo bem característico decorre da análise, por exemplo, do parâmetro coliformes totais, que no período dos 10 anos considerados, apresentam incidências da análise fora do padrão compreendidas entre $2,07 \%$ a $86,1 \%$, ou seja, uma variação muito significativa, dado que tais valores correspondem a índices dos anos de 2012 e 2011 respectivamente. 0 mesmo comportamento pode ser observado nos índices analisados para os parâmetros cloro residual e turbidez.

Em vista disso, a forma de utilização dos recursos hídricos e o despejo de substâncias tóxicas nos corpos d'água têm-se demandado constantes estudos de monitoramento e atenção especial dos gestores governamentais (MARQUES et al., 2007).

Aliado a este problema, há um crescente aumento da carga orgânica lançada nos mananciais principalmente superficiais, derivado da ausência de tratamento do esgoto e do lançamento de esgoto à rede de drenagem pluvial, o que demanda aumentos significativos da presença de microrganismos no meio hídrico. A grande preocupação decorre de possíveis contaminações também aos mananciais subterrâneos, que são amplamente utilizados como fonte de abastecimento à grande parte da população do estado do Maranhão.

A necessidade de se prover um tratamento adequado e de se garantir a potabilidade da água destinada ao abastecimento público, em qualquer ponto do sistema de abastecimento, decorre de se ter garantia dos índices dos parâmetros analisados dentro da conformidade, ou seja, dentro dos padrões estabelecidos na Portaria de Consolidação n 5, Anexo XX de 2017 do Ministério da Saúde (BRASIL, 2017), atualmente vigente no país.

Embora o PLANSAB procure manter a premissa de deslocar o tradicional foco dos planejamentos clássicos em saneamento básico, pautados na hegemonia de investimentos em obras físicas, para um melhor balanceamento destas medidas estruturantes há necessidade de um fortalecimento dessas ações entendidas como um melhor suporte político e gerencial dos prestadores de serviço. Somente desta forma, há de se garantir melhor qualidade dos dados e dos serviços ofertados à população do Estado.

\section{CONCLUSÃO}

Os indicadores analisados, apesar de serem importantes, não são potencialmente consistentes quando considerados para avaliação, uma vez que não são confiáveis. A metodologia empregada pelo SNIS ainda é muito incipiente não permitindo uma análise profunda, uma vez que não apresenta todos as informações da série histórica selecionada para o estudo. 
Ressalta-se que os indicadores de qualidade da água selecionados neste estudo são de fundamental importância, dando-se destaque ao parâmetro de turbidez, pois apesar de não apresentar risco sanitário efetivo, este dificulta a detecção dos microrganismos

\section{REFERÊNCIAS}

BRASIL. Lei no 11.445, de 5 de janeiro de 2007. Institui as diretrizes nacionais para o saneamento básico e a Política Federal de Saneamento Básico no Brasil. Diário Oficial da União, Brasília, 2007.

BRASIL, Ministério da Saúde. Portaria de Consolidação n0 5, de 28 de setembro de 2017. Consolidação das normas sobre as ações e os serviços de saúde do Sistema Único de Saúde. Diário Oficial da União, 2017

IBGE - Instituto Brasileiro de Geografia e Estatística. Censo demográfico, 2010. Disponível em: https://www.ibge.gov.br/estatisticas/sociais/populacao/9662-censo-demografico-2010. html?=\&t=resultados. Acesso em: 13 fev. 2019

IBGE - Instituto Brasileiro de Geografia e Estatística. Projeção demográfica, 2018. Disponível em: https://www.ibge.gov.br/apps/populacao/projecao/. Acesso em 13 fev. 2019

CORTON, M. L. Benchmarking in the Latin American Water Sector: the case of Peru. Util. Policy, v. 11, n. 3, p. 133-142, 2003.

MARQUES, M. N. et al. Avaliação do impacto da agricultura em áreas de proteção ambiental, pertencentes à bacia hidrográfica do rio Ribeira de Iguape, São Paulo. Quím. Nova, v. 30, n. 5, p. 1171-1178, 2007.

SNIS - Sistema Nacional de Informações sobre Saneamento. Diagnóstico dos Serviços de Água e Esgotos, 2017. Brasília. Disponível em: http://www.snis.gov.br/diagnostico-anual-agua-e-esgotos/ diagnostico-ae-2017. Acesso em: 5 mar. 2019.

VEIGA, T. B. et al. Análise de Indicadores Propostos Para a Gestão de Resíduos Sólidos Urbanos no Brasil. In: Convibra - Administração,10, 2013, SP. Anais..., São Paulo, 2013.

VON SPERLING, M. Introdução à qualidade das águas e ao tratamento de esgotos. 2. ed. Belo Horizonte: UFMG, 1996. 


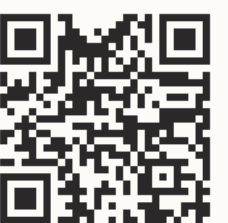

A autenticidade desse artigo pode ser conferida no site https://periodicos. set.edu.br
1 Mestre em Tecnologia Ambiental; Professora na Universidade CEUMA - UniCEUMA. E-mail: marylin.farias@gmail.com

2 Doutora em Engenharia Civil; Professora na Universidade Federal do Maranhão - UFMA.

E-mail: solangeboni57@gmail.com

3 Mestre em Sustentabilidade de Ecossistemas; Professora na Universidade CEUMA - UniCEUMA.

E-mail: prof.nathaliapinheiro@gmail.com

4 Mestre em Engenharia Civil; Professor da Universidade CEUMA - UniCEUMA. E-mail: rcboni71@gmail.com

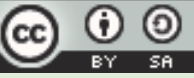

Este artigo é licenciado na modalidade acesso abertosob a Atribuição-Compartilhalgual CC BY-SA

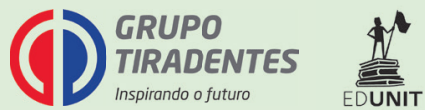

